\title{
Análisis bibliométrico de estudios sobre factores socioeconómicos en estudiantes universitarios
}

\section{Lilia Esther Palacio Sprockel}

https://orcid.org/0000-0002-6836-4009 Universidad de La Guajira, Colombia liliapalacio@uniguajira.edu.co
Javier Dionicio Vargas Babilonia

https://orcid.org/0000-0002-0096-127X Universidad de La Guajira, Colombia jvargas@uniguajira.edu.co

\section{Stella Lucila Monroy Toro}

https://orcid.org/0000-0002-1823-0213

Universidad de La Guajira, Colombia

smonroy@uniguajira.edu.co

\section{Resumen}

El artículo presenta una actualización de las variables socioeconómicas relacionadas con el abandono de los estudios universitarios. Se utilizó la metodología del metaanálisis, a partir de la revisión sistemática de estudios científicos relacionados con el tema. De 143 artículos publicados en el período 2005-2019, se seleccionaron 71 que cumplieron plenamente con los criterios de selección de la muestra: relación directa con la temática del estudio, publicados en revistas en español del área de educación e indexadas en bases de datos especializadas de acceso abierto. El presente estudio corrobora las incidencias de las variables socioeconómicas y sociodemográficas como causales del bajo rendimiento académico y de la consecuente deserción estudiantil; uno de los factores socioeconómicos preponderantes resultó ser la escolaridad del padre o la madre. También muestra que la finalidad de las investigaciones sobre las variables socioeconómicas se orienta principalmente a la formulación de políticas universitarias para disminuir el abandono estudiantil.

\section{Palabras clave (Fuente: tesauro de la Unesco)}

Análisis bibliográfico; bibliometría; educación universitaria; enseñanza superior; estudiante universitario; deserción escolar; variables socioeconómicas. 


\title{
Bibliometric Analysis of Studies on Socioeconomic Factors in University Students
}

\begin{abstract}
The article introduces an update of the socioeconomic variables related to university dropping out. The meta-analysis method is used based on the systematic review of scientific studies on the subject. Of 143 articles published during 2005-2019, 71 were selected that fully met the sample selection criteria: directly related to the subject matter, published in education journals in Spanish, and indexed in specialized open-access databases. This study corroborates the effect of socioeconomic and sociodemographic variables as causes of poor academic performance and consequent dropping out; a predominant socioeconomic factor turned out to be the education level of the father or mother. It also demonstrates that the purpose of research into socioeconomic variables is mainly oriented towards the formulation of university policies to reduce dropping out.
\end{abstract}

\section{Keywords (Source: Unesco thesaurus)}

Bibliographic analysis; bibliometrics; higher education; university education; university students; college students; dropping out; socio-economic variables. 


\section{Análise bibliométrica de estudos sobre fatores socioeconômicos em estudantes universitários}

Resumo

O artigo apresenta uma atualização das variáveis socioeconômicas relacionadas com o abandono dos estudos universitários. Utilizou-se a metodologia da meta-análise, a partir da revisão sistêmica de estudos científicos relacionados com o tema. De 143 artigos publicados no período 2005-2019, selecionaram-se 71 que cumpriram plenamente os critérios de seleção da amostra: relação direta com a temática do estudo; publicados em revistas em espanhol da área de educação, indexadas em bancos de dados especializados de acesso aberto. O presente estudo corrobora as incidências das variáveis socioeconômicas e sociodemográficas como causais do baixo rendimento acadêmico e da consequente evasão estudantil; um dos fatores socioeconômicos preponderantes resultou sendo a escolaridade do pai ou da mãe. Também mostra que a finalidade das pesquisas sobre as variáveis socioeconômicas é orientada principalmente para a formulação de politicas universitárias para diminuir o abandono estudantil.

\section{Palavras-chave (Fonte: tesauro da Unesco)}

Análise bibliográfica; bibliometria; formação universitária; ensino superior; estudante universitário; evasão escolar; variáveis socioeconômicas. 
La propuesta de esta investigación presenta diferencias metodológicas con respecto a las investigaciones exploradas, debido a que no se encontraron en la muestra de documentos estudios bibliométricos con metaanálisis, metodología que para Botella y Zamora (2017) es una herramienta adecuada para la investigación educativa, pues se caracteriza por la precisión, la objetividad y la replicabilidad, lo cual marca una diferencia con respecto a las numerosas investigaciones que se han elaborado sobre esta temática. Los estudios evidencian que los factores socioeconómicos han sido uno de los principales criterios que se han analizado para determinar la causa del bajo rendimiento académico y la deserción de estudiantes universitarios. No obstante, es importante resaltar que en los estudios concertados, se observa que la sistematización de la categoría "factores socioeconómicos" conlleva a una amplia subcategorización que no sigue una tendencia uniforme, sino que, por el contrario, se presentan de manera diversa y con escasas similitudes.

El objetivo de esta investigación es actualizar el enfoque de estos estudios, explorar si siguen enfocándose puntualmente en la correlación de estas variables o han emergido nuevas variables y correlaciones, así como determinar avances hacia publicaciones con propuestas educativas más factibles. Se explora así la contribución de estas investigaciones de acuerdo con los objetivos de la investigación y su alcance, ya que se considera que hay suficientes resultados científicos para trascender a investigaciones de un nivel mayor, como por ejemplo las investigaciones aplicadas.

El análisis bibliométrico presentado es el resultado de desarrollar las fases propias de un proceso de metaanálisis haciendo una revisión exhaustiva de los contenidos de las investigaciones para dar respuestas a los objetivos propuestos. La revisión sistemática ha permitido representar gráficamente las categorías codificadas, pudiendo determinar nuevas experiencias en el campo de este tipo de estudio y análisis de correlación entre los factores socioeconómicos y su efecto sobre variables de interés académico.

\section{El problema de investigación}

La apertura de las universidades como un medio para expandirse y cumplir su misión de acceso a la educación, abriendo sus fronteras hacia las poblaciones de estudiantes de familias más vulnerables, no ha sido un hecho aislado, a sabiendas de que la educación es uno de los ejes principales para el desarrollo y crecimiento de un país (Unesco, 2013). No obstante, el crecimiento exponencial de la matrícula de estudiantes de bajo nivel socioeconómico en el sistema de educación superior ha sido punto de discusión, dado que, hoy por hoy, realmente se contempla como un fenómeno complejo (Donoso y Schiefelbein, 2007).

Específicamente en América Latina, la apertura a la educación superior no ha sido suficiente para que la educación sea un derecho para todos los ciudadanos, tal y como lo dispone la Unesco cuando resalta las graves brechas internas en esta región, donde los factores sociales, como la "clase social, la condición indígena y la zona de residencia, son los vectores principales por donde pasan dichas inequidades" (2013, p. 17). De tal modo, estas condiciones pesan tanto sobre los más desfavorecidos, aunque logren avanzar en su educación, nunca se comparan con el desarrollo de la población más aventajada.

En estos planteamientos y miradas en busca de que la educación alcance a todos por igual, es claro que tales realidades y situaciones solo se ponen de manifiesto cuando los estudiantes de bajos niveles económicos deben enfrentarlas, sorteando primero los criterios de ingreso académicos y administrativos $\mathrm{y}$, durante el período de formación, los reglamentos de permanencia y continuidad para mantenerse activos en las estructuras educativas.

Una diversidad compleja de fuerzas externas e internas poco flexibles actúan sobre los sistemas universitarios, como pasa con los sistemas de admisión, los programas basados en los méritos para la continuidad y los de obtención de becas, así como la formulación de los reglamentos institucio- 
nales, los cuales demandan una alta exigencia académica para los estudiantes que desean participar de estos beneficios. Sobre todo porque para este conjunto de estudiantes ellos representan una opción para mantenerse en las universidades, lo cual es todo un reto para aquellos que no cuentan con una mínima condición social, económica y académica necesaria para la continuidad y finalización de sus estudios superiores.

De acuerdo con un reporte de Unesco (2006), el éxito de los estudiantes en la educación superior estaría directamente relacionado con su desempeño en las pruebas de admisión y el tipo de establecimiento de enseñanza secundaria, elementos que a su vez se relacionan con el $\mathrm{ni}$ vel socioeconómico de origen de los estudiantes. (Carrasco et al., 2014, p. 97)

Definitivamente, esta nota confirma que el estatus económico de la familia de los estudiantes dependientes actúa como uno de los factores incidentes del desempeño académico. La Organización para la Cooperación Económica y el Desarrollo (OCDE, 2018) en su informe sobre el Panorama de la Educación afirma que el nivel social sigue siendo el factor principal que influye sobre la participación en la educación y el aprendizaje de los estudiantes universitarios.

Esta realidad social que determina el estatus económico familiar, aunada a las condiciones estructurales de las universidades, son factores que soportan la posibilidad de la continuidad de los estudiantes en los estudios universitarios hasta su titulación. Para autores como Cruz et al. (2014) y Garbanzo (2013), en investigaciones que analizan los factores asociados al rendimiento académico, es inusual encontrar abordajes que contemplen el nivel socioeconómico en estudiantes universitarios; no obstante, este componente se considera importante en su repercusión y un insumo para la formulación de políticas universitarias orientadas a la inclusión estudiantil.
Cabe resaltar también que, a pesar de todos los esfuerzos y estrategias para aumentar las tasas de retención de estudiantes en las universidades a nivel mundial, el índice de deserción de los estudiantes sigue siendo una de las mayores preocupaciones en el ámbito educativo. Según Ariza et al. (2013), los más recientes informes publicados por la OCDE advierten que para el periodo entre 2008 y 2011, el porcentaje de estudiantes que desertó de las universidades, tanto públicas como privadas osciló entre el $44,9 \%$ y el $56 \%$, cifras que ubican a Colombia, Italia, México, Chile, entre otros, como los países con mayor índice de deserción.

El caso de Argentina lo describen García (2014) y García y Adrogué (2015) en cuanto a: bajas tasas de graduación según los tiempos de finalización; problemas de rendimiento estudiantil y abandono, y esfuerzos realizados mediante políticas públicas para subsanar estas realidades. Una situación similar se presenta en Chile, donde -de acuerdo con los análisis de una investigación cualitativa que realizó Flanagan (2017), para determinar las causas de la deserción escolar, a partir de las experiencias de un grupo de estudiantes indígenas de universidades públicas y privadas- los mismos estudiantes reconocen que su estatus socioeconómico no les hubiera permitido siquiera ingresar ni mucho menos permanecer en la universidad, y también resaltan los problemas de las instituciones para ofrecerles el apoyo requerido hasta terminar sus estudios.

De acuerdo con estos datos y resultados, actualmente se observa que es necesario redoblar los esfuerzos para mejorar la equidad en la educación, con el objetivo de aumentar el porcentaje de estudiantes que concluyen el nivel superior. En el informe de la OCDE (2018), se certifica la urgencia de aumentar los niveles de titulación en educación terciaria, debido a que actualmente "el cambio tecnológico, la digitalización y la innovación conceden un gran valor a las competencias avanzadas, al tiempo que los empleos menos cualificados están siendo suprimidos del mercado" (s.p.). Por lo tanto, 
la profesionalización sigue siendo uno de los caminos que garantiza el crecimiento y desarrollo de los pueblos del mundo.

\section{Metodología de análisis documental}

El objetivo principal de este estudio es presentar una actualización de la utilización de las variables socioeconómicas que afectan la continuidad y la permanencia en la formación de estudiantes universitarios. No obstante, en forma indirecta se busca descubrir las características de las propuestas o recomendaciones que los autores han realizado para minimizar esta situación problemática. Para lograr estos objetivos se llevó el proceso de revisión sistemática de acuerdo con la metodología del metaanálisis, la cual consiste en "el análisis cuantitativo de revisiones de la literatura científica sobre una pregunta específica" (Botella y Zamora, 2017, p. 20).

Se revisaron específicamente artículos de revistas científicas publicadas durante el período 2005-2019 y para ello se utilizó: el buscador especializado Google Scholar; las bases de datos de La Red y del Sistema Regional de Información en Línea de Revistas Científicas de América Latina y el Caribe, España y Portugal, Redaly c y Latindex, respectivamente; la Biblioteca Electrónica Scielo; el Índice Bibliográfico Nacional de Colciencias (Publindex) y de la base de datos bibliográfica de la Universidad de La Rioja (Dialnet); y se eligieron las revistas en español de acceso abierto especializadas en el área de educación.

Los trabajos de investigación seleccionados fueron aquellos que presentaron como descriptores o palabras clave los factores socioeconómicos, variables sociodemográficas, factores sociodemográficos, perfil socioeconómico, nivel socioeconómico y estudiantes universitarios. Se exceptuaron los estudios de esta naturaleza aplicados especialmente a población de estudiantes universitarios en condición especial o discapacitados, así como aquellos cuyos resultados no fueran orientados al área educativa.
La primera exploración se hizo mediante la lectura de los títulos en conjunto con las palabras clave y los descriptores, lo que arrojó una preselección de 143 artículos (después de aplicarle los filtros a las bases de datos antes mencionadas). La segunda revisión implicó una lectura de los resúmenes y la población de estudio y, en algunos casos, una lectura más profunda de los artículos, ya que el criterio de búsqueda al respecto de las variables sociodemográficas, en una significativa parte de los trabajos, no se ubicó en las palabras clave, pero sí en el resumen y/o en el desarrollo del contenido del escrito; finalmente la población de análisis se conformó en 71 artículos científicos.

Con la información de los artículos revisados se prosiguió a la fase de codificación de los estudios, para lo cual se construyó una tabla de datos metaanalítica producto de la lectura de los contenidos, hasta organizar la información de referencia en varias categorías, de acuerdo con el objetivo de la investigación y los hallazgos encontrados durante la revisión sistemática. La base de datos organizada de esta forma, según Botella y Zamora (2017), constituyó el material con el que se trabajó en la siguiente fase, que corresponde al análisis e interpretación de los resultados.

\section{Análisis estadístico de los estudios primarios}

A continuación se presentan los datos generales de la información extraída de los artículos científicos como producto de la revisión metaanalítica. Para la representación general de los datos e información se construyeron gráficas utilizando una Hoja de Excel de Office 2007, y en los casos donde se consideró necesario se utilizaron tablas de doble entrada para hacer más fácil la presentación de los mismos. De cada una de estas representaciones se hará una interpretación de los resultados, para finalmente hacer observaciones más relevantes con base en la muestra de documentos analizados. La Figura 1 representa las estadísticas de la producción de investigaciones en el contexto de las universi- 
dades de los países relacionados. En ella se puede observar que Colombia lidera este bloque de países con 29,6\% de la muestra de estudio, seguida por México con $12,7 \%$, España y Costa Rica con $11,3 \%$, Chile con $8,5 \%$, Argentina con $7 \%$ y Perú con $5,63 \%$, mientras que Ecuador, El Salvador, Venezuela, Uruguay y Nicaragua aportan menos del 3\% de la producción total sistematizada.

\section{Figura 1. Producción académica por país}

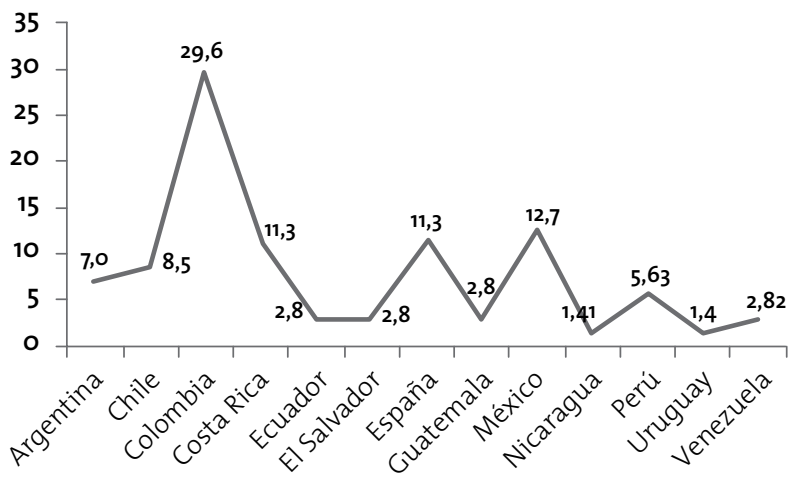

Fuente: elaboración propia.

La Figura 2 refleja la producción por año en el período de estudio (2005-2019), donde se puede observar que 2014 fue un año rico en este tipo de estudios sobre la temática investigada, con un $22,5 \%$, mientras que en 2017 desarrolló un 18,3\% de los productos analizados, en 2016 un 14,1\% y en 2013 un $11,3 \%$. Los demás años se encuentran por debajo del 10\% de la producción científica.

Figura 2. Producción académica por año

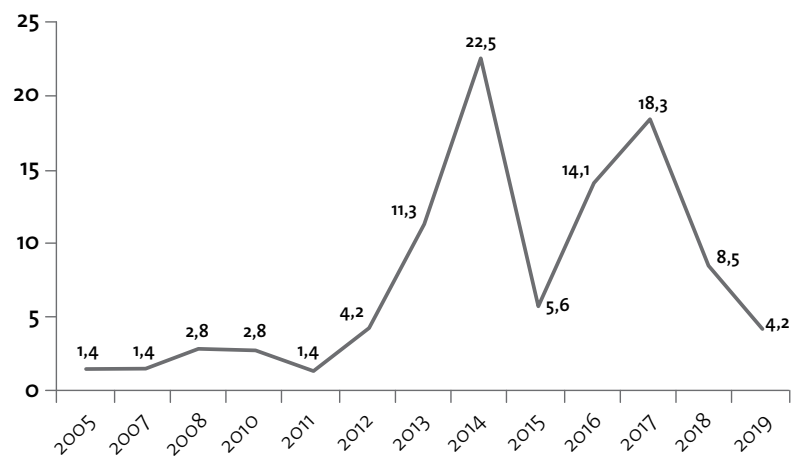

Fuente: elaboración propia.
Es importante observar que la producción científica arrojó mayor proporción de estudios enfocados en universidades oficiales o públicas, muy por encima de las instituciones privadas. Con superioridad en las universidades autónomas, oficiales o públicas, se realizaron los estudios sobre los factores socioeconómicos y su posible influencia en el rendimiento académico de los estudiantes universitarios y la deserción escolar. Aproximadamente una cuarta parte de los estudios se realizaron en universidades privadas, un número menor en otras instituciones y organismos y, en mínima proporción, hubo estudios en ambos modelos de universidades (públicas y privadas).

Así mismo, se observa que todas las investigaciones en las universidades privadas, del total de la muestra de estudios primarios seleccionados, se ubican en el período 2013-2019. Es de señalar que estas investigaciones en universidades privadas fueron de tipo mixto, es decir, cuantitativo-cualitativo, con solo una exclusivamente cualitativa, mientras que la mayoría de los estudios en las universidades públicas se orientaron por criterios exclusivamente empírico-analíticos basados principalmente en el análisis estadístico descriptivo e inferencial. No obstante, se localizó un mínimo de investigaciones cualitativas y de categoría mixta donde se integra la metodología cuantitativa con la cualitativa.

En países como Argentina, Costa Rica, España, México, Nicaragua y Venezuela, los estudios se concentraron en universidades públicas principalmente; en El Salvador, se encuentra que sus estudios fueron en universidades privadas, mientras que Costa Rica, Chile y Perú han realizado investigaciones en los dos tipos de universidad. En cuanto a la metodología utilizada, se obtuvo que $78,9 \%$ fueron investigaciones cuantitativas, $12,7 \%$ de origen mixto cuantitativo-cualitativo, $7 \%$ del tipo cualitativo y $1,4 \%$ sin método de investigación observable.

En cuanto a las metodologías cuantitativas, prevaleció el diseño no experimental, descriptivo, de 
campo, transversal y correlacional. Por lo general, el análisis de los datos se hizo a través de la estadística descriptiva e inferencial mediante el software SSPS, con los modelos de regresión lineal y multifactorial. En las investigaciones cualitativas utilizaron la metodología del interaccionismo simbólico y estudios fenomenológicos. En cuanto a las investigaciones de enfoque mixto, solo una utilizó la investigación fenomenológica, la hermenéutica y el interaccionismo simbólico. El 5,7\% especificó que utilizaron la técnica de los grupos focales y todas hicieron entrevistas en profundidad como instrumentos de recolección de información.

La investigación de La Red et al. es el único trabajo de este grupo de estudios que describe un modelo basado en técnicas de Data Warehouse (DW) y Data Mining (DM), para determinar los perfiles de rendimiento académico. El DM "utiliza los métodos de Inteligencia Artificial y del Aprendizaje de Máquinas para extraer patrones y tendencias de forma que posibiliten la descripción y comprensión de los datos y también la predicción de comportamientos futuros" (2016, p. 71).

Otra de las categorías emergentes que se puede extraer del análisis estadístico es la finalidad de la investigación, de la cual emergieron los indicadores que se observan en la Figura 3. La importancia práctica de mayor interés es que para 31\% de las investigaciones hay necesidad de tomar en consideración los resultados para un cambio en las políticas universitarias. Un 18,3\% de las investigaciones tuvieron como justificación atender la deserción estudiantil o el abandono escolar y 19,7\% apuntó su alcance a la hora de mejorar el rendimiento académico. Las políticas públicas y los programas de retención ocuparon cada uno el $7 \%$ del interés de las investigaciones.

La categoría "Otros" con 9,9\%, se refiere a aquellos trabajos que fueron muy específicos en cuanto a su finalidad, como por ejemplo los que midieron la correlación de la dependencia a las drogas y el bienestar psicológico con las variables sociodemográfi- cas. En lo que se refiere a la importancia de atender los factores socioeconómicos en relación con los beneficios del estudio para la inserción laboral, el emprendimiento, la profesionalización, la creación de una cooperativa de ahorro y el riesgo de exclusión social y laboral, obtuvieron el 1,4\% de interés de las investigaciones.

\section{Figura 3. Finalidades de las investigaciones}

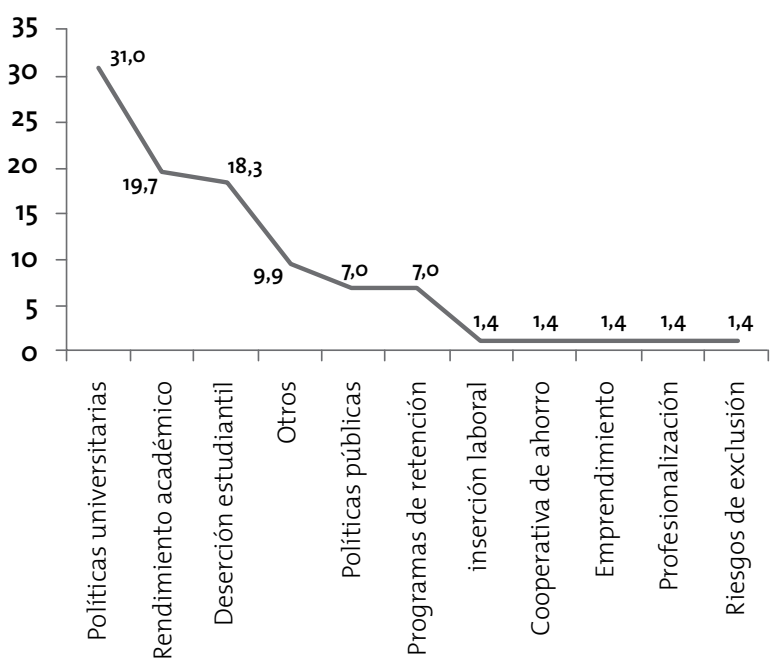

Fuente: elaboración propia.

\section{Análisis crítico de las investigaciones}

El análisis crítico se hizo a partir del método hermenéutico, que da cuenta de los hallazgos sustentados en los objetivos de investigación. En la Figura 4 (abajo) se observa una relación que reúne de forma concisa los aspectos más relevantes encontrados en la revisión de los documentos. Como se puede constatar, las recomendaciones de investigación se proyectan hacia las políticas universitarias, las cuales abordan una serie de lineamientos y programas institucionales, como programas de retención y permanencia, bolsas de trabajo, financiamiento, becas, planes de tutoría y nivelación, como parte de los factores institucionales que son competencia de las universidades para minimizar el efecto de los factores socioeconómicos de la población estudiantil de menores recursos. 
La mayoría de las propuestas no tienen ni diseño ni configuración planificada y han sido elaboradas como recomendaciones con base en los resultados, en la experiencia de los investigadores y en las acciones que han tomado otras universidades. Salvo en algunos casos, se dieron propuestas abiertas a estudios de factibilidad, tales como la investigación de La Red et al. (2016) con la propuesta tecnológica para manejar gran cantidad de información, y la de Vargas et al. (2018), que propone conformar una cooperativa de ahorro y crédito, con sustento en los argumentos derivados de la investigación sobre los efectos de la variables sociodemográficas como factores de riesgo para la permanencia de los estudiantes universitarios en la Universidad de la Guajira.

El estudio de Hernández et al. (2016) fue el único que propuso el diseño de un sistema de alertas tempranas (SAT), que toma en cuenta los factores socioeconómicos como posibles causas del bajo rendimiento académico, el abandono y la deserción escolar, y contempla las cuatro fases de un sistema de esta naturaleza -medición, generar alerta, revisión profesional del caso y plan de contingencia-, totalmente adaptado al componente académico de las universidades. Cabe destacar que ambos estudios se aplicaron en el contexto de dos universidades colombianas.

Sevilla et al. (2010) pusieron a operar varias estrategias, clasificadas en económicas, académicas e institucionales, es decir, que se corresponden con las variables estudiadas según el modelo de Tinto (1975 citado por González et al., 2007). Los resultados de la investigación de Díaz et al. (2017) muestran que algunas de las estrategias académicas, específicamente la que se refiere a las tutorías, ya implementadas en universidades españolas, no han sido lo suficientemente exitosas, no porque no hayan tenido efectividad, sino porque la proporción de estudiantes que acuden a las tutorías es baja con respecto a la muestra, pero son efectivas, porque, con respecto a los estudiantes que asistieron a las tutorías, el 57\% alcanzó un rendimiento medio y $10 \%$ un rendimiento alto.
La Figura 4 es una representación gráfica de los aspectos más relevantes de las investigaciones en función de la asociación de la deserción y del rendimiento académico, como fuente teórica y metodológica para la formulación de las políticas universitarias. Ella señala las coincidencias analíticas de la repercusión de las variables descriptivas socioeconómicas en los planes y programas estratégicos de retención y permanencia estudiantil.

En lo que se refiere a las técnicas de análisis de los datos, la mayoría de trabajos se orienta al tipo de investigación cuantitativa, descriptiva y correlacional mediante con fundamento en la estadística descriptiva e inferencial. Las técnicas estadísticas que caracterizan a estos documentos prácticamente coinciden con la investigación de Fonseca y García (2016), en cuanto a que los estudios analíticos de los datos recogidos dan cuenta de la utilización de técnicas estadísticas complejas que incluyen desde correlaciones hasta regresiones logísticas y análisis factoriales.

Las técnicas estadísticas que mayormente se utilizaron se orientaron precisamente a detectar posibles relaciones entre variables, es decir, a determinar la dependencia de una variable en función de las variables independientes, tales como los modelos de regresión lineal (correlación de Pearson), modelos de regresión logística y regresión multinomiales, regresión lineal múltiple, pruebas de análisis de la varianza univariantes (Anova), análisis multivariado de la varianza (Manova). En menor medida, fueron utilizadas técnicas como regresiones múltiples por mínimos cuadrados ordinarios (MCO), análisis de clúster, análisis de correspondencia múltiple y análisis discriminante. Estos análisis estadísticos quedan debidamente justificados al observar la heterogeneidad en las categorizaciones tanto de las variables dependientes como de las independientes, que se requieren para medir el comportamiento y establecer las posibles relaciones de causalidad que las vinculan. 


\section{Figura 4. Aspectos relevantes de las investigaciones}

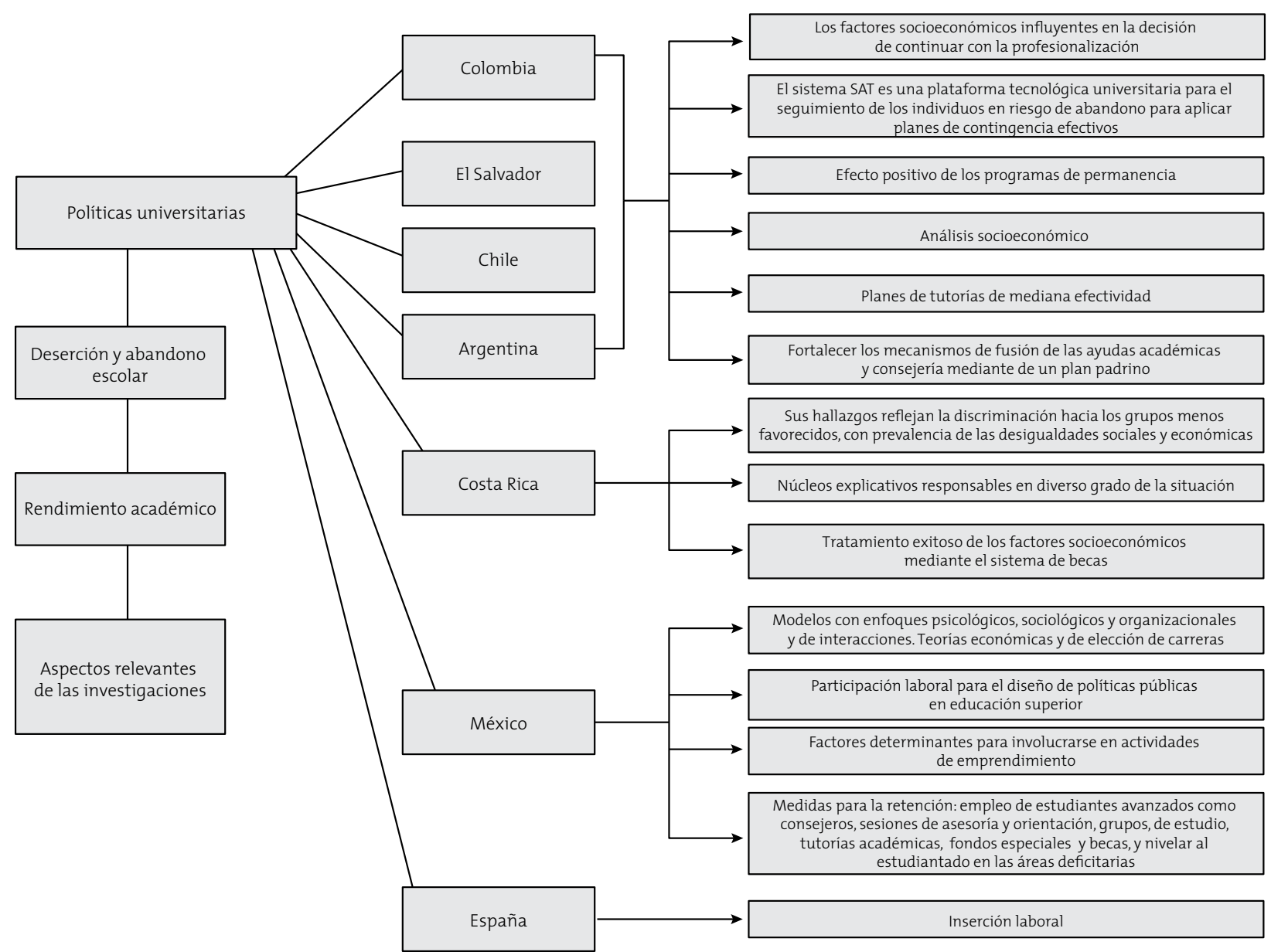

Fuente: elaboración propia.

Las investigaciones de Barrero et al. (2015), Contreras (2017), Fernández (2013), Garbanzo (2013), Ramírez (2014), Rodríguez y Hernández (2008), Ruiz (2018), Souza et al. (2017) y Velázquez y González (2017) determinaron que los factores socioeconómicos influyen escasamente o no influyen en el rendimiento o la deserción estudiantil, en comparación con otros factores, como los académicos, institucionales y los individuales. En cambio, otros estudios analizados indican que los factores socioeconómi-

$1 \quad$ Entre los principales: Barahona et al. (2016), Canales (2016), Chan et al. (2017), Coschiza et al. (2016), Fontalvo et al. (2015), cos, como variable independiente, sí influyen en los estudios de deserción.

Autores como Barahona et al. (2016), Carvajal et al. (2018), Fontalvo et al. (2015), Gardner et al. (2016) y Ruiz (2018) confirman en sus investigaciones que la deserción es un fenómeno multicausal donde los factores socioeconómicos influyen tanto o en igual

Franco y Romero (2017), García y Adrogué (2015), González et al. (2007), González (2015), González y Pedraza (2017), González et al. (2017), Hernández et al. (2016), Mayer y Cerezo (2018), Ríos et al. (2014), Rodríguez et al. (2017), Rodríguez et al. (2016), Sevilla et al. (2010), Vargas et al. (2018) y Vergara y Perea (2014). 
medida que los otros factores personales, académicos e institucionales. El estudio de Barahona et al. (2016) tomó directamente la deserción como consecuencia de uno de los predictores académicos más sobresalientes, como lo es el rendimiento académico.

En la revisión se observó que hay una variedad de modos de hacer operativas las variables que intervienen en este tipo de estudios. No obstante, para seguir uno de los modelos teóricos más reconocidos y usados en la literatura sobre estas temáticas, se estableció el modelo de Tinto (1975, citado por González et al., 2007) de la Figura 5 (abajo), en la que se observan las relaciones con los factores individuales, factores institucionales, factores socioeconómicos y factores académicos con los fenómenos de deserción universitaria y rendimiento académico.

En menor medida, en otras investigaciones se categorizaron factores de riesgo y factores de protección, variables de sujeto, variables de contexto, variables preuniversitarias (Fernández y Silva (2014) y núcleos explicativos (Rodríguez y Hernández, 2008). En menor medida, fueron tomadas en cuenta las variables ambientales, sociodemográficas y biográficas. Las variables dependientes mantuvieron ciertamente homogeneidad, siendo las más constantes: deserción estudiantil o escolar y rendimiento académico; permanencia, persistencia, abandono y/o desvinculación. Otras variables dependientes, como inserción laboral, riesgo de exclusión social y laboral, profesionalización, emprendimiento y cooperativa, se pueden considerar como emergentes de este estudio.

Entre las variables significativas menos estudiadas estuvieron los comportamientos de riesgo (trabajo sin contrato, fraude académico, ensayos clínicos, donación de semen u órganos, tráfico de drogas y prostitución) en los que han incurrido para obtener dinero y poder pagar los costos de los estudios. En consecuencia, los resultados sugieren una asociación entre el nivel económico y el riesgo social, lo que haría de la universidad una puerta para la exclusión y no para la inclusión.
Estas actividades aparecían en muestras de estudiantes del último semestre, lo cual indica que constituyen una alternativa social riesgosa en extremo para obtener la titulación, sobre todo en especialidades que son muy costosas cuyos requisitos para graduarse exigen de mayor disponibilidad económica, tales como son las pasantías y las tesis de grado, y otros gastos en los que es necesario incurrir para estar solventes con la universidad.

Como aspectos claves a resaltar en cuanto a la diversidad de las variables inmersas en este tipo de estudios y su consecuente análisis de causalidad está que la mayor parte de las investigaciones no presentaron las razones por las cuales pusieron a operar las variables independientes y dependientes. Se pudo observar en ciertos casos que los investigadores propusieron una categorización en función de los datos que recibieron de las propias universidades; en otros casos, siguieron el patrón de otras investigaciones sobre la misma temática; también se observaron casos en función del conocimiento sobre la materia y, en menor proporción, se encontraron los artículos científicos que sustentaron la categorización y la subcategorización con los modelos teóricos explicativos del abandono escolar.

No todas las investigaciones hacen alusión explícita a los modelos teóricos con los cuales analizaron las tasas de permanencia y abandono. Es por ello que se toman los modelos representativos de aquellas investigaciones que sí ofrecieron la información en la cual apoyaron la puesta en operación de las variables e indicadores. Por ello se observa en los párrafos siguientes las fuentes secundarias y terciarias según los hallazgos de la revisión de los artículos en cuestión.

En este último caso, las investigaciones que midieron las variables de estudio en función de los modelos teóricos que analizan los fenómenos de permanencia y abandono se corresponden con las de Fonseca y García (2016), González et al. (2007), González y Pedraza (2017) y Velázquez y González (2017), 
soportados en teorías psicológicas, sociológicas, económicas, organizacionales e interaccionistas. Entre los modelos teóricos, uno de los más referidos ha sido el de Tinto (1975, citado por Fonseca y García, 2016); sin embargo, hay otros modelos también importantes, como el de Bean y Metzner (1985, citados por Fonseca y García, 2016). Por su parte, González et al. (2007) señalan tres modelos explicativos del abandono: el adaptativo e integracionista, el estructuralista y el economicista.

Antes de resumir los diferentes modelos encontrados en la revisión de bibliográfica, se presentan las teorías que subyacen a estos modelos, como lo son los enfoques presentados por Fonseca y García (2016), cuando se refieren a los postulados que identifican las teorías psicológicas, sociológicas, económicas, organizacionales e interaccionistas.

Tabla 1. Postulados teóricos de permanencia y abandono de estudios universitarios

\begin{tabular}{|c|l|}
\hline Teorias & \multicolumn{1}{|c|}{ Postulados } \\
\hline Psicológicas & $\begin{array}{l}\text { Identifican los rasgos de personalidad de } \\
\text { los estudiantes que se titulan respecto } \\
\text { de los que no lo hacen. No toman en } \\
\text { cuenta el impacto del contexto en el } \\
\text { comportamiento del estudiante. }\end{array}$ \\
\hline Sociológicas & $\begin{array}{l}\text { Analizan el impacto de los fenómenos } \\
\text { sociales en el abandono, sin considerar } \\
\text { la influencia de las características } \\
\text { institucionales. }\end{array}$ \\
\hline Económicas & $\begin{array}{l}\text { Responden principalmente a la relación } \\
\text { costo-beneficio de los estudios. Los } \\
\text { factores económicos pueden influir en la } \\
\text { decisión de abandonar la institución. }\end{array}$ \\
\hline Organizacionales & $\begin{array}{l}\text { Identifican las características de la } \\
\text { institución universitaria (servicios que } \\
\text { ofrece a los estudiantes) que explican la } \\
\text { permanencia y el eventual abandono de } \\
\text { los estudiantes en la universidad. }\end{array}$ \\
\hline Interaccionistas & $\begin{array}{l}\text { Teorías que relacionan las características } \\
\text { de los estudiantes y el tipo de entorno } \\
\text { ofrecido por la institución. }\end{array}$ \\
\hline
\end{tabular}

Fuente: Fonseca (2013, citado por Fonseca y García, 2016).

Para estudiar los modelos es fundamental tener en consideración las teorías psicológicas, sociológi- cas, económicas, organizacionales e interaccionistas esbozadas de forma general en la Tabla 1, mientras en la Tabla 2 se presentan las fuentes teóricas que soportan las variables independientes que pueden influir en el abandono estudiantil, materializadas en factores individuales o personales, académicos, institucionales y, por supuesto, socioeconómicos.

Tabla 2. Diferentes perspectivas de las teorias y modelos del abandono

\begin{tabular}{|c|l|}
\hline Autor/año & \multicolumn{1}{|c|}{ Modelos explicativos } \\
\hline $\begin{array}{c}\text { González et al. } \\
\text { (2007) }\end{array}$ & $\begin{array}{l}\text { Adaptativo e integracionista, } \\
\text { estructuralista y economicista. }\end{array}$ \\
\hline $\begin{array}{c}\text { Cabrera et al. } \\
\text { (2006b, citados } \\
\text { por González y } \\
\text { Pedraza, 2017) }\end{array}$ & $\begin{array}{l}\text { Adaptativo, estructuralista, } \\
\text { economicista, psicopedagógico y } \\
\text { ecológico. }\end{array}$ \\
\hline $\begin{array}{c}\text { Fonseca y García } \\
\text { (2016) }\end{array}$ & $\begin{array}{l}\text { Características familiares y personales, } \\
\text { habilidades y capacidades, experiencias } \\
\text { preuniversitarias. }\end{array}$ \\
\hline $\begin{array}{c}\text { Velázquez y } \\
\text { González (2017) }\end{array}$ & $\begin{array}{l}\text { Enfoque psicológico, enfoque } \\
\text { sociológico, teorías económicas, } \\
\text { modelos organizacionales y de } \\
\text { interacciones y las teorías de elección } \\
\text { de carrera. }\end{array}$ \\
\hline
\end{tabular}

Fuente: elaboración propia.

Como se puede observar en la presentación que hace González et al., cada modelo se asocia con su respectiva teoría. En lo que se refiere al modelo adaptativo e integracionista, este proviene de la teoría de la persistencia que tiene como base las teorías sobre integración académica y social, mientras que el modelo estructuralista "centra su explicación del fenómeno del abandono en las contradicciones que poseen los diferentes subsistemas político, económico, social y cultural que conforman la sociedad" (2007, p. 73). El modelo economicista, que integra los factores socioeconómicos, relaciona el abandono con la falta de correspondencia entre la inversión que hace el estudiante -en variables como tiempo, energía y recursos-y los beneficios que obtiene. 
Tabla 3. Características de los modelos del abandono universitario

\begin{tabular}{|c|c|}
\hline Teoria/modelo & Postulados \\
\hline Adaptativa & $\begin{array}{l}\text { Desadaptación entre el sujeto y la cultura } \\
\text { universitaria a la que se incorpora. }\end{array}$ \\
\hline Estructural & $\begin{array}{l}\text { Contradicciones de los diferentes } \\
\text { subsistemas que influyen en el individuo. }\end{array}$ \\
\hline Economicista & $\begin{array}{l}\text { Alternativa de invertir tiempo, energía } \\
\text { y recursos para producir futuros } \\
\text { beneficios superiores respecto a costos } \\
\text { en educación. }\end{array}$ \\
\hline Psicopedagógica & $\begin{array}{l}\text { Explicaciones del abandono por las } \\
\text { características psicológicas y personales } \\
\text { del estudiante. }\end{array}$ \\
\hline Ecológica & $\begin{array}{l}\text { Los sujetos se encuentran inmersos en } \\
\text { un ambiente ecológico, compuesto de } \\
\text { contextos inmediatos o externos que } \\
\text { influyen en su desarrollo. }\end{array}$ \\
\hline
\end{tabular}

Fuente: Cabrera et al. (2006b, citados por González y Pedraza, 2017).
Barrero et al. (2015) y Sevilla et al. (2010) han formulado una alternativa basada "en el enfoque de la psicología educativa para solventar la multicausalidad, en la cual se puedan considerar estos modelos y sus postulados para controlar las razones que provocan la deserción o abandono universitario". Una solución bastante compleja es tratar de religar todos estos fundamentos teóricos, gnoseológicos y epistemológicos para diseñar una alternativa que impacte favorablemente en la disminución de los índices de abandono y deserción en las universidades.

En línea con los objetivos de la investigación, estos cruces y combinaciones producen una información integrada, por cuanto interesan las variables de deserción y rendimiento y su aporte a la formulación de políticas universitarias o públicas. En la Figura 5 se resaltan las principales relaciones e incidencias mediante un mapa topológico en correspondencia con los objetivos de esta investigación.

Figura 5. Correlación de los factores académicos y no académicos con el rendimiento académico y la deserción estudiantil

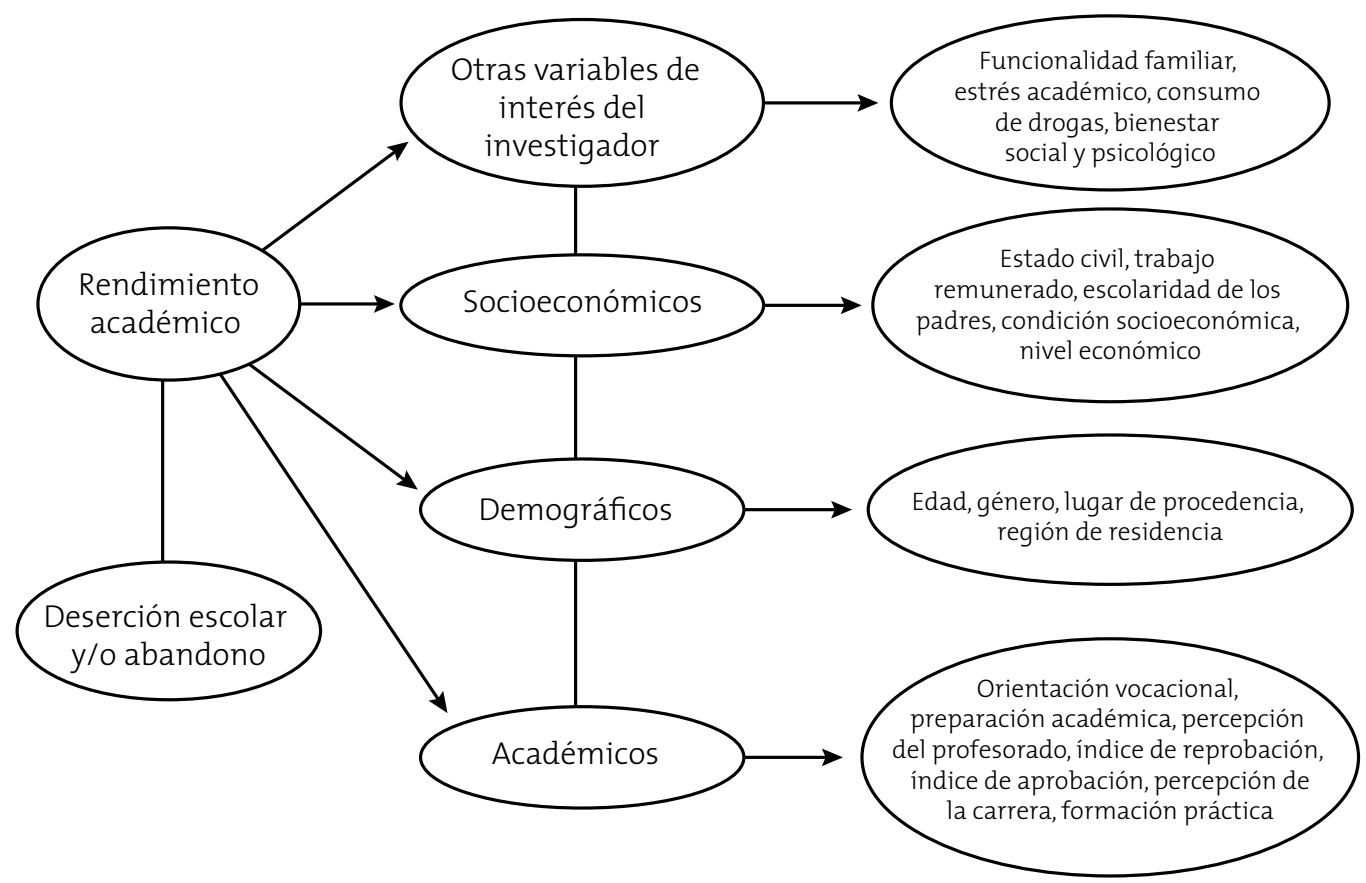

Fuente: elaboración propia. 
Volviendo a las estadísticas de la Figura 3 (arriba), para autores como Coschiza et al. (2016), Flanagan (2017), Fonseca y García (2016), Garbanzo (2013), Gartner et al. (2016), La Red et al. (2016), Repreza (2013) y Rodríguez y Hernández (2008), entre otros, la formulación de políticas universitarias reviste el mayor interés y está por encima de la deserción, el rendimiento y las políticas públicas, o sea que para los investigadores consultados uno de los aspectos medulares para confrontar y disminuir los índices de deserción y aumentar el rendimiento académico es la formulación de políticas universitarias, como uno de los principales factores institucionales que competen directamente a las organizaciones universitarias.

En la Figura 5 se muestran los factores o macrovariables utilizados con mayor frecuencia en los estudios analizados. Se puede observar la operatividad de las dimensiones o subcategorías, punto de coincidencia de los autores, ligado a las recomendaciones para el desarrollo del marco de políticas que las universidades pueden implementar para contener el riesgo de recurrencia del abandono escolar. Es de anotar que en el 90\% de los casos se consideran indicadores demográficos como la edad, el género y lugar de procedencia. En cuanto a las variables socioeconómicas, las más estandarizadas en las encuestas y cuestionarios fueron: estado civil, condición laboral y nivel económico familiar, junto con la escolaridad de la madre o el padre, como el mayor predictor del abandono. Estas convergencias indican que los factores sociales y económicos tienen serias repercusiones en la decisión de los estudiantes de interrumpir o abandonar los estudios universitarios.

En lo que respecta a los factores académicos, sí se encontró variedad de subdimensiones e indicadores ligados a la particularidad, exclusividad y autonomía en la elaboración de los reglamentos institucionales de cada universidad analizada, aunque se debe notar que ciertas variables académicas, según los modelos teóricos del abandono, aparecen con cierta consistencia en todas las investigaciones, sobre todo en las que miden el rendimiento previo o el conocimiento adquirido en la etapa anterior a los estudios terciarios, así como los resultados de las pruebas de ingreso.

Los artículos de Armenta et al. (2008), Díaz et al. (2017), Fernández et al. (2011), García (2014), Hernández (2018), Mora et al. (2019) y Elvira-Valdés y Pujol (2012), entre otros, tienden a determinar el rendimiento estudiantil en función del grado de afectación de las variables académicas y socioeconómicas, e incluso ofrecen nuevos modelos estratégicos para mejorar el conocimiento de los estudiantes. En lo que respecta a los estudios llevados a cabo para determinar las causas del bajo rendimiento académico, casi siempre se lo relaciona con la deserción o abandono escolar.

Los autores para los cuales la deserción de los estudiantes universitarios es el fenómeno que se debe atender prioritariamente fueron Abarca y Sánchez (2005), Barahona et al. (2016), Barrero et al. (2015), Carvajal et al. (2018), Cock (2014), Ríos et al. (2014), Sevilla et al. (2010) y Zamora (2019). En sus hipótesis, el abandono escolar está asociado a la influencia de los factores socioeconómicos y el rendimiento académico.

En lo que se refiere a las variables que causan la deserción estudiantil, según los trabajos analizados, las variables más preponderantes son: edad, género y nivel socioeconómico, y se encuentran significativas coincidencias con estas variables, tanto que han sido tomadas como derivadas de otras de mayor espectro y abstracción. En cuanto al rendimiento académico las variables más predominantes son las socioeconómicas y las académicas. Un caso especial lo representa el estudio de Ruiz (2018) aplicado a la educación a distancia, con conclusiones apropiadas sobre el peso de factores personales como los que más tienen efecto en la decisión de desertar, entre los que se destaca la falta de tiempo para el estudio, seguido de indicadores como los viajes, motivos familiares y cambio de prioridades. 
En los trabajos de investigación de Franco y Romero (2017) y García y Méndez (2017) se relacionaron las variables socioeconómicas con factores de exclusión laboral o empleabilidad que puedan llevar a continuar o no con los estudios profesionales. Los datos de estos estudios demuestran que las variables socioeconómicas influyen en la decisión de continuar o no con la formación profesional. Por ello se destaca la importancia de factores de empleabilidad y su influencia en la decisión de mejorar las condiciones laborales. Las coincidencias de estos con otros estudios permiten resaltar que el ingreso familiar es uno de los principales factores socioeconómicos que motiva a los estudiantes a persistir hasta lograr la profesionalización.

\section{Reflexiones propias}

Los resultados del análisis bibliométrico de este conglomerado de investigaciones dan soporte epistemológico, teórico, metodológico y práctico a la utilidad y aporte de las investigaciones, tanto a la universidad como a las políticas públicas gubernamentales, en las cuales la universidad es un actor estructural fundamental e irremplazable, sin olvidar que las universidades son uno de los principales actores en la formulación de políticas públicas y que estos estudios representan una fuente de conocimiento científico para su actuación en el campo del diseño y aplicación de estos lineamientos, a favor del mejoramiento de la formación y bienestar de las sociedades.

En las universidades que manejan un significativo número de matrícula queda demostrado que los factores económicos y la condición social de los estudiantes influyen en su rendimiento, lo que hace posible manejar sistemas universitarios enfocados a trabajar para cambiar esta realidad social o, por lo menos, intentarlo, ahora que se cuenta con datos e información de calidad que sustentan las políticas universitarias y públicas, aunque evidentemente el mayor impacto lo tendrán las políticas públicas y su correcta aplicación, seguimiento y control.
En pleno siglo XXI, a pesar de la apertura de los programas y de los recursos para integrar en un principio de equidad a toda la población, todavía persisten la diferenciación, la discriminación y las desigualdades sociales, lo que se refleja en el alto impacto de la condición social en los porcentajes y niveles de rendimiento académico y deserción escolar.

A pesar de todos los esfuerzos de los gobiernos y de las instituciones de educación superior por superar esta brecha de conocimiento en los menos favorecidos, aún siguen siendo los grupos en condiciones económicas más estables los que más permanecen en aulas, los que menos abandonan la formación, los que más persisten hasta lograr la titulación y los que más aportan al crecimiento y desarrollo de un país. Esta situación de desigualdades sociales no es un problema que puedan resolver las universidades de forma aislada, sino un asunto complejo, estructural y transversal donde están relacionados el Estado, los gobiernos y la sociedad civil, para los que la universidad es un ente mediador.

Con base en estas consideraciones, las universidades interesadas en diseñar e implementar efectivos programas de permanencia y retención de alto impacto en su gestión académica requieren cambios estratégicos para favorecer verdaderamente la solución anticipada a los problemas de deserción estudiantil, empezando por viabilizar de forma autónoma el análisis de los modelos teóricos y prácticos del abandono universitario y seleccionar el que más se adapte a su realidad institucional, para hacer luego los ajustes y cambios en función de su particularidad institucional.

Para elegir el modelo teórico que mejor se adapte a su presupuesto y organización, en las universidades es importante concebir estos análisis internos de manera más anticipada y continua; es decir, hay que determinar las condiciones sociales de los estudiantes antes de iniciar su formación universitaria y las estadísticas de los que no se inscribieron después de culminar un período académico. Es muy probable 
que este escenario se pueda lograr implementando mecanismos que favorezcan el desarrollo de investigaciones prospectivas basadas en las proyecciones y tendencias sobre las tasas de abandono, reprobación y permanencia.

Otro aspecto interesante a resaltar es el de la saturación en la base de datos de investigaciones cuantitativas sobre las incidencias de las variables socioeconómicas y sociodemográficas, referidas tanto a la deserción como al rendimiento académico en el estadio universitario, las cuales han mantenido sus resultados a pesar del tiempo y de los análisis actualizados con sistemas tecnológicos. En este sentido, las investigaciones de metodología mixta, es decir, las investigaciones cuantitativas-cualitativas, pudieran ser una opción viable para la obtención de resultados más cohesionados y coherentes de acuerdo con los modelos teóricos existentes, ya que la participación de los propios estudiantes ofrecería un cúmulo de información importante que no es posible recolectar con las encuestas y cuestionarios.

El Estado es el primer interesado en conocer estas cifras, sobre todo en el caso de las universidades oficiales, debido a que el presupuesto y la inversión social en la educación tienen una partida importante de los ingresos económicos nacionales, presupuesto e inversión que se estarían perdiendo. Por ello estos estudios adquieren gran relevancia y arrojan verdaderos resultados para optimizar los procesos de planificación y control.

Independientemente de las causas de la deserción, del abandono o del bajo rendimiento académico, ya sea por variables socioeconómicas, académicas, psicológicas, ambientales o demográficas, contribuir a mejorar la condición social y económica de los estudiantes universitarios siempre será una alternativa para minimizar los casos de abandono e interrupción temporal o definitiva de los estudios superiores. Las condiciones académicas definitivamente son responsabilidad de la institución educativa, y como los factores sociales en gran medida son exter- nos a la universidad, al constituir la coyuntura estructural, es el Estado quien debe asumir su cuota de responsabilidad en el soporte económico de la población vulnerable. Incluso los factores socioeconómicos son externos a la universidad. De hecho, es la universidad la que media entre esa realidad y el Estado.

Es importante acotar que obtener el grado de profesionalización genera un impacto positivo en el desarrollo de un país, o sea, que la profesionalización está relacionada con el nivel de calidad de vida, siendo mayor para aquellos que terminan sus carreras que para aquellos que la abandonan. Estas inferencias se consideran válidas, pues miden el éxito del modelo de desarrollo económico de los países, así como la estructura y estabilidad de su condición política, social, económica y cultural. Así, aquellos que no desarrollan ninguna competencia de desempeño profesional, porque se desvincularon en forma permanente de la universidad, tendrán comprometido su bienestar económico. Lo dicho hasta acá es coherente con el hallazgo de que es en los países con mayores desigualdades sociales donde se dan mayores porcentajes de deserción, comparados con aquellos que tienen una economía más estable, como se observa en Latinoamérica.

Un aspecto preocupante es que se requiere de un equilibrio de ambos géneros para el desarrollo de un país, insertos ambos en el campo profesional y laboral de forma equilibrada. Ese desbalance de talento humano que se sesga hacia un mismo sexo podría incluso arriesgar la renovación de la vida en el planeta. Ante esta situación, si los estudios siempre arrojan resultados no equilibrados, y no es posible establecer una tendencia, eso dificulta los modelos de políticas públicas y, por ende, las políticas universitarias. Es este un aspecto objeto de discusión, al que los estamentos educativos del nivel superior deben prestar atención, sobre todo en los países del tercer mundo o subdesarrollados, ya que les costará más aún muchos años poder entonces alcanzar el crecimiento y desarrollo por medio de la investigación y la innovación. 
En cuanto a la implementación de políticas institucionales para la retención, permanencia y graduación o titulación de los estudiantes universitarios, no se logra reducir significativamente la deserción y el abandono, aunque, por el volumen de investigaciones, se pueda afirmar que las universidades están cumpliendo su compromiso de índole social; sin embargo, es una realidad que son ineficaces los esfuerzos por reducir las brechas entre las matrículas de ingreso y las de egreso, lo que genera inquietudes y las siguientes interrogantes: los presu- puestos para los planes y proyectos educativos con estos fines ison suficientes para el total de estudiantes que necesitan el apoyo económico?, ¿será que se derivan los recursos para estudiantes que en verdad no los necesitan?, ¿se deberá medir la satisfacción y la eficacia de estas políticas con otros indicadores?, ¿se hace el debido control y seguimiento a las políticas ejecutadas?, ¿será que la insuficiencia e ineficacia de las políticas viene de que la situación económica y de vulnerabilidad tiene a otras fuentes estructurales que escapan al control de las universidades?

\section{Referencias}

Abarca, A. y Sánchez, M. (2005). La deserción estudiantil en la educación superior: el caso de la Universidad de Costa Rica. Actualidades Investigativas en Educación, 5(especial), 1-22. https://revistas.ucr.ac.cr/index.php/ aie/article/view/9186/17625

Ariza, C., Torres, L.y Varela, O. (2013). Pertinencia de las acciones para la prevención de la deserción universitaria: caso Universidad Santo Tomás, seccional Bucaramanga periodo 2008-2011. Revista Temas, 7, 11-28. https:// doi.org/10.15332/rt.voi7.571

Armenta, N., Pacheco, C. y Pineda, E. (2008). Factores socioeconómicos que intervienen en el desempeño académico de los estudiantes universitarios de la Facultad de Ciencias Humanas de la Universidad Autónoma de Baja California. Revista de Investigación en Psicologia, 11(1), 153-165. https://dialnet.unirioja.es/servlet/ articulo?codigo $=2747347$

Barahona, P., Veres, E.y Aliaga, V. (2016). Deserción académica de la Universidad de Atacama, Chile. Comuni@cción, 7(2), 27-37. https://dialnet.unirioja.es/servlet/articulo?codigo=5755757

Barrero, F., Barrero, C., Borja, H. y Montaño, M. (2015). Factores de riesgo asociados a la deserción estudiantil universitaria en programas de pregrado de la Universidad de San Buenaventura, Bogotá (2009-2013). Revista Academia y Virtualidad, 8(2), 60-72. https://dialnet.unirioja.es/servlet/articulo?codigo $=5236378$

Botella, J. y Zamora, Á. (2017). El meta-análisis: una metodología para la investigación en educación. Educación XX1, 20(2), 17-38. https://doi.org/10.5944/educXX1.19030

Canales, A. (2016). Diferencias socioeconómicas en la postulación a las universidades chilenas. El rol de factores académicos y no académicos. Calidad en la Educación, 44, 129-157. https://dialnet.unirioja.es/servlet/ articulo?codigo $=5614777$

Carrasco, E., Zúñiga, C. y Espinoza, J. (2014). Elección de carrera en estudiantes de nivel socioeconómico bajo de universidades chilenas altamente selectivas. Revista Calidad en la Educación, 40, 95-128. https://www.cned. cl/otros-estudios/eleccion-de-carrera-en-estudiantes-de-nivel-socioeconomico-bajo-de-universidades 
Carvajal, C., González, J. y Sarzoza, S. (2018). Variables sociodemográficas y académicas explicativas de la deserción de estudiantes en la Facultad de Ciencias Naturales de la Universidad de Playa Ancha (Chile). Formación Universitaria 11(2), 3-12. http://dx.doi.org/10.4067/S0718-50062018000200003

Chan, E., Morales, L., Ruiz, J. y Vaca, J. (2017). Factores sociodemográficos asociados a la victimización delictiva en estudiantes universitarios de tres ciudades mexicanas. Pensamiento Psicológico, 15(2), 93-107. https://doi. org/10.11144/Javerianacali.PPSI15-2.fsav

Cock, A. (2014). Identificación de factores asociados a la deserción de estudiantes universitarias en condición de madres solteras. [Trabajo de investigación] Resumen Analítico en Educación, Universidad Católica de Colombia.

Contreras, C. (2017). Rendimiento académico de los alumnos de último año de licenciaturas presenciales e ingeniería de la Facultad Multidisciplinaria de llobasco durante el ciclo I-2017. Anuario de Investigación, 7 , 125-139. http://www.diyps.catolica.edu.sv/wp-content/uploads/2018/og/10RendimientosAN2018.pdf

Coschiza, C., Fernández, J., Redcozub, G., Nievas, M. y Ruiz, H. (2016). Características socioeconómicas y rendimiento académico. El caso de una universidad argentina. REICE. Revista Iberoamericana sobre Calidad, Eficacia y Cambio en Educación, 14(3), 51-76. https://doi.org/10.15366/reice2016.14.3.003

Cruz, Z., Medina, J., Vázquez, J., Espinosa, E. y Antonio, A. (2014). Influencia del nivel socioeconómico en el rendimiento académico de los alumnos del programa educativo de Ingeniería Industrial en la Universidad Politécnica de Altamira. En N. Carmona, Y. Santamaría y L. Almanza (coords.), Ciencias Administrativas y Sociales. Handbook T-V. (pp. 24-38). Congreso Interdisciplinario de Cuerpos Académicos - ECoRfan. https:// dialnet.unirioja.es/servlet/articulo?codigo $=4887110$

Díaz, L. M., Sarmiento, H.y Rodríguez, D. J. (2017). Relación entre el rendimiento académico en Matemáticas y los estilos de aprendizaje de los estudiantes de la Fundación Universidad Autónoma de Colombia. Revista de Estilos de Aprendizaje, 10(20), 34-62. http://revistaestilosdeaprendizaje.com/article/view/1056

Donoso, S.y Schiefelbein, E. (2007). Análisis de los modelos explicativos de retención de estudiantes en la universidad: una visión desde la desigualdad social. Estudios Pedagógicos, 33(1), 7-27. http://dx.doi.org/10.4067/ So718-07052007000100001

Elvira-Valdés, M. y Pujol, L. (2012). Autorregulación y rendimiento académico en la transición secundaria-universidad. Revista Latinoamericana de Ciencias Sociales, Niñez y Juventud, 10(1). http://revistaumanizales. cinde.org.co/rlcsnj/index.php/Revista-Latinoamericana/article/view/612

Fernández-Molina, M., González, V.y Del Molino, G. (2011). Perfil del alumnado universitario de Educación Infantil. Un estudio descriptivo desde los inicios del Espacio Europeo de Educación Superior hasta los estudios de grado (2006-2010). Revista de Investigación Educativa, 29(1), 187-203. http://www.redaly c.org/articulo. $\mathrm{oa} ? \mathrm{id}=283322813011$

Fernández, N. (2013). Perfil del estudiante que persiste en el contexto de la Universidad Simón Bolívar. Educere, 17(58), 501-508. http://www.redaly c.org/articulo.oa?id=35630404013 
Fernández, X. y Silva, E. (2014). Deserción estudiantil universitaria en el primer semestre. El caso de una institución de educación superior ecuatoriana. En F. Ceballos (ed.), Deserción, calidad y reforma universitaria (1 ed., pp.34-48). Cuadernos del Contrato Social por la Educación.

Flanagan, A. (2017). Experiencias de estudiantes de primera generación en universidades chilenas: realidades y desafíos. Revista de la Educación Superior, 46(183), 87-104. http://dx.doi.org/10.1016/j.resu.2017.06.003

Fonseca, G. y García, F. (2016). Permanencia y abandono de estudios en estudiantes universitarios: un análisis desde la teoría organizacional. Revista de la Educación Superior, 45(179), 25-39. http://dx.doi.org/10.1016/j. resu.2016.06.004

Fontalvo, W., Castillo, M. y Polo, S. (2015). Análisis multivariado para determinar los factores más relevantes de deserción estudiantil presentes en el programa de Ingeniería Industrial de una Universidad del Caribe colombiano. Prospect, 13(1), 86-99. http://dx.doi.org/10.15665/rp.v13i1.363

Franco, M. y Romero, G. (2017). Identificación de factores socioeconómicos y de empleabilidad en la decisión de continuar con la profesionalización (un caso aplicado para 5 programas de tecnologías de la UNIAJC). Sotavento, 29, 42-53. http://dx.doi.org/10.18601/01233734.n29.05

Garbanzo, G. (2013). Factores asociados al rendimiento académico en estudiantes universitarios desde el nivel socioeconómico: Un estudio en la Universidad de Costa Rica. Revista Electrónica Educare, 17(3), 57-87. https://www.redaly c.org/pdf/1941/194128798005.pdf

García, A. (2014). Rendimiento académico y abandono universitario: Modelos, resultados y alcances de la producción académica en la Argentina. RAES. Revista Argentina de Educación Superior, 6(8), 9-38. http://repositorio.cedes.org/handle/123456789/3217

García,A.y Adrogué,C.(2015).Abandono de los estudios universitarios:dimensión,factores asociadosydesafíos para la política pública. Revista Fuentes, 16, 85-106. https://dialnet.unirioja.es/servlet/articulo?codigo=5159610

García, D. y Méndez, J. (2017). Estudiantes universitarios en riesgo de exclusión social: una aproximación a una realidad social oculta. Revista de la Asociación de Sociología de la Educación, 10(1), 37-49. http://dx.doi. org/10.7203/RASE.10.1.8996

Gardner, L., Dussán, C. y Montoya, D. (2016). Aproximación causal al estudio de la deserción en la Universidad de Caldas. Periodo 2012-2014. Revista Colombiana de Educación, 70, 319-340. https://doi.org/10.17227/012039 16.70rce 319.340

González, E. (2015). Factores de contexto socioeconómicos y educativos en estudiantes de nivel superior, sugerencia para una realidad actual. Interamerican Journal of Psychology, 49. https://www.redaly c.org/articulo.oa?id=28446020010

González, M., Álvarez, P., Cabrera, L. y Bethencourt, J. (2007). El abandono de los estudios universitarios: factores determinantes y medidas preventivas. Revista Española de Pedagogía, 236, 71-86. https://revistadepedagogia.org/wp-content/uploads/2007/06/236-07.pdf 
González, T. y Pedraza, I. (2017). Variables sociofamiliares asociadas al abandono de los estudios universitarios. Educatio Siglo XXI, 35(2), 365-388. http://dx.doi.org/10.6018/j/298651

Hernández, J., Rojas, M. y López, Y. (2016). Caracterización del desertor y diseño del sistema de alertas tempranas: Facultad de Minas, Universidad Nacional de Colombia. Revista Logos, Ciencia \& Tecnología, 8(1), 77-87. http://www.redaly c.org/articulo.oa?id=517754055009

La Red, D., Karanik, M., Giovannini, M., Báez, M.y Torre, J. (2016). Descubrimiento de perfiles de rendimiento estudiantil: un modelo de integración de datos académicos y socioeconómicos. Campus Virtuales, 5(2), $70-83$. https://dialnet.unirioja.es/servlet/articulo?codigo $=5696008$

Mayer, L.y Cerezo, L. (2018). ¿Quiénes se fueron? Aproximaciones a los motivos de abandono de un programa de apuntalamiento a las trayectorias educativas de estudiantes universitarios. Actualidades Investigativas en Educación, 18(2), 1-30. https://doi.org/10.15517/aie.v18i2.33160

Mora Bravo, L., Reinoso, J., Serrano, C., Rodríguez, D. y Larriva, D. (2019). Influencia de las variables socio-demográficas en el rendimiento académico de los estudiantes de la Facultad de Ciencias Médicas de la Universidad de Cuenca - Ecuador. Ateneo, 21(1), 51-62. http://colegiomedicosazuay.ec/ojs/index.php/ateneo/ article/view/84

OCDE (2018). La OCDE sostiene que es necesario redoblar los esfuerzos para mejorar la equidad en la educación. OCDE - Mejores políticas para una vida mejor. [Portal web] https://www.oecd.org/centrodemexico/medios/laocdesostienequeesnecesarioredoblarlosesfuerzosparamejorarlaequidadenlaeducacion.htm

Ramírez, C. (2014). Factores asociados al desempeño académico según nivel de formación pregrado y género de los estudiantes de educación superior Colombia. Revista Colombiana de Educación, 66, 201-222. http:// revistas.pedagogica.edu.co/index.php/RCE/article/view/2594/2434

Repreza, E. (2013). Causas de deserción en estudiantes de la Universidad Católica de El Salvador. Anuario de Investigación, 2, 35-55. http://www.diyps.catolica.edu.sv/wp-content/uploads/2016/05/2DesercionAnVol2.pdf

Ríos, R., Peña, R. y Aguilar M. (2014). Factores predisponentes de abandono en estudiantes de Medicina de la Facultad de Ciencias Médicas de la Universidad de San Carlos de Guatemala. [Ponencia] Cuarta Conferencia Latinoamericana sobre el Abandono en la Educación Superior. Congreso CLABES IV, Medellín, Colombia.

Rodríguez, J. y Hernández, J. (2008). La deserción escolar universitaria en México. La experiencia de la Universidad Autónoma Metropolitana Campus Iztapalapa. Actualidades Investigativas en Educación, 8(1), 1-30. https://doi.org/10.15517/AIE.V811.9308

Ruiz, M. (2018). Factores que influyen en la deserción de los alumnos del primer ciclo de educación a distancia en la Escuela de Administración de la Universidad Señor de Sipán. Períodos académicos 2011-1 al 20131: lineamientos para disminuir la deserción. Educación, 27(52), 161-173. https://doi.org/10.1880o/educacion.201801.009 
Sevilla, D., Puerta, V.y Dávila, J. (2010). Influencia de los factores socioeconómicos en la deserción estudiantil de la Carrera de Ciencias Sociales. Ciencia e Interculturalidad, 6(1), 72-84. https://dialnet.unirioja.es/servlet/ articulo?codigo $=6576527$

Souza, M., Zea, A., Rodríguez, G. y Molina, A. (2017). Estilo de vida y factores socioeconómicos en estudiantes de electivas de actividad física y deporte de la Pontificia Universidad Javeriana. Análisis, 49(90), 229-243. https://dialnet.unirioja.es/servlet/articulo?codigo $=6140729$

Unesco (2013). Situación Educativa de América Latina y el Caribe: Hacia la educación de calidad para todos al 2015. Oficina Regional de Educación para América Latina y el Caribe (orealc/unesco Santiago). Ediciones del Imbunche.

Vargas, J., Monroy, S. y Arregocés, I. (2018). Análisis socioeconómico de los estudiantes para el montaje e implementación de una cooperativa de aporte y crédito en la Universidad de la Guajira, sede principal Riohacha. Boletín Redipe, 7(11), 206-219. https://dialnet.unirioja.es/servlet/articulo?codigo=6729075

Velázquez, Y. y González, M. (2017). Factores asociados a la permanencia de estudiantes universitarios: caso UAMм-Uat. Revista de la Educación Superior, 46(184), 117-138. https://doi.org/10.1016/j.resu.2017.11.003

Vergara, M. y Perea, D. (2014). Deserción en estudiantes del Programa Plan Estudia de una universidad privada de Manizales, Caldas-Colombia. 2008-2009. Ánfora, 21(37), 61-80. http://www.redaly c.org/articulo. oa?id=357833888003

Zamora-Araya, J. (2019). Abandono en estudiantes de la carrera en Enseñanza de la Matemática de la UNA, cohorte 2017. Descripción de sus principales características sociodemográficas y académicas. [Memorias] I Congreso Internacional de Ciencias Exactas y Naturales, Universidad Nacional, Costa Rica, http://dx.doi. org/10.15359/cicen.1.6 
